\title{
Cellular senescence: friend or foe to respiratory viral infections?
}

\author{
William J. Kelley ${ }^{1,2,3}$, Rachel L. Zemans ${ }^{1,2,4}$ and Daniel R. Goldstein (1) 1,2,3 \\ Affiliations: ${ }^{1}$ Dept of Internal Medicine, University of Michigan, Ann Arbor, MI, USA. ${ }^{2}$ Program in Immunology, \\ University of Michigan, Ann Arbor, MI, USA. ${ }^{3}$ Dept of Microbiology and Immunology, University of Michigan, \\ Ann Arbor, MI USA. ${ }^{4}$ Program in Cellular and Molecular Biology, University of Michigan, Ann Arbor, MI, USA.
}

Correspondence: NCRC B020-209W, 2800 Plymouth Road, Ann Arbor, MI 48104, USA. E-mail: drgoldsta umich.edu

@ERSpublications

Senescence associates with fibrotic lung diseases. Emerging therapies to reduce senescence may treat chronic lung diseases, but the impact of senescence during acute respiratory viral infections is unclear and requires future investigation. https://bit.ly/2SAPpx6

Cite this article as: Kelley WJ, Zemans RL, Goldstein DR. Cellular senescence: friend or foe to respiratory viral infections? Eur Respir J 2020; 56: 2002708 [https://doi.org/10.1183/13993003.02708-2020].

ABSTRACT Cellular senescence permanently arrests the replication of various cell types and contributes to age-associated diseases. In particular, cellular senescence may enhance chronic lung diseases including COPD and idiopathic pulmonary fibrosis. However, the role cellular senescence plays in the pathophysiology of acute inflammatory diseases, especially viral infections, is less well understood. There is evidence that cellular senescence prevents viral replication by increasing antiviral cytokines, but other evidence shows that senescence may enhance viral replication by downregulating antiviral signalling. Furthermore, cellular senescence leads to the secretion of inflammatory mediators, which may either promote host defence or exacerbate immune pathology during viral infections. In this Perspective article, we summarise how senescence contributes to physiology and disease, the role of senescence in chronic lung diseases, and how senescence impacts acute respiratory viral infections. Finally, we develop a potential framework for how senescence may contribute, both positively and negatively, to the pathophysiology of viral respiratory infections, including severe acute respiratory syndrome due to the coronavirus SARS-CoV-2. 


\section{Cellular senescence: definition and evolutionary origins}

Cellular senescence describes a state of permanent replicative arrest in normally proliferative cells. Originally discovered in vitro in human fibroblasts by HAYFLICK and co-workers $[1,2]$, the concept of cellular senescence was met with controversy. Initially, some investigators were sceptical and believed that senescence was an in vitro artefact. But with accumulating in vivo evidence, including the presence of senescence in aged human skin cells [3], scientists accepted senescence as a true biological phenomenon. Senescence is now known to contribute to a variety of age-related diseases including type 2 diabetes, obesity, atherosclerosis, COPD, pulmonary fibrosis and others [4-6]. With ageing, our cells continually divide, shorten their telomeres and accumulate mutations and DNA damage, all of which ultimately increase the likelihood of oncogenesis $[7,8]$.

Under typical physiological conditions, oncogene-induced senescence may hinder the development of certain cancers including lymphoma [9] and prostate cancer [10] and improve patient response to chemotherapeutics [11]. In addition, senescent cells may upregulate phagocytic receptors, therefore increasing phagocytosis and immune surveillance to reduce cancer development. Additionally, senescent cells secrete extracellular matrix proteins and growth factors that contribute to some restorative processes including wound healing [12]. Similarly, senescent cells have been shown to reduce liver $[13,14]$ and pancreatic fibrosis and promote wound healing [15]. Furthermore, fibroblasts made senescent by the matricellular protein CCN1 limit fibrosis in cutaneous wounds [16]. Senescent cells may contribute to tissue growth during embryonic development $[17,18]$. In summary, senescence probably evolved to respond to the acute stress of organ development, mitigate against the acute effects of damaged cells to return tissue to homeostasis, and ultimately reduce the development of cancer [19].

With improvements in public health in the 20th century and subsequent extensions of the human lifespan, senescence has emerged as a contributing factor to several chronic age-associated diseases. Indeed, we now recognise senescence as one of the key hallmarks of ageing, as senescence occurs as we age naturally [20]. Although a key hallmark of ageing, senescence is not sufficient for all ageing phenotypes [20], and senescence can occur in young hosts as a result of acute stressors (see next section). However, the importance of senescence in acute inflammatory conditions, especially respiratory viral infections, is less well appreciated. Here, we first briefly review how senescence contributes to chronic inflammation, particularly in the context of lung diseases, and emerging therapies to treat senescence. Then, we discuss the role senescence plays in acute respiratory viral infections and implications for such a role during infection with severe acute respiratory syndrome coronavirus 2 (SARS-CoV-2).

\section{What leads to senescence and the resulting senescence-associated secretory phenotype?}

Senescence can arise from both repeated cellular division and cellular stressors. Replicative senescence arises from repeated cellular divisions, which occur naturally when we age. Stress-induced senescence arises from cellular stressors such as increases in reactive oxygen species (ROS), which correlate with DNA damage [21, 22]. These can result from irradiation, chemotherapeutic agents, chronic exposure to pollutants (cigarette smoke), exposure to pathogens and some forms of accelerated ageing syndromes, such as progeria [23-26]. Therefore, cells from both young and aged hosts can exhibit senescence. As previously mentioned, senescence is one of the key biological hallmarks of ageing and contributes to ageing via inflammation, tissue exhaustion and impaired stem-cell renewal [20].

Importantly, stem cells can become senescent as we age. Specifically, mesenchymal stem cells isolated from older humans (aged $>70$ years) exhibit senescence including reduced proliferation and a chronic inflammatory secretome, which impairs haematopoietic stem and progenitor cell (HSPC) replication and increases HSPC monocyte chemoattractant protein-1 and interleukin (IL)-8 expression [27]. Consequently, senescent mesenchymal stem cells cannot be used in stem-cell therapeutics due to their inability to proliferate and their reduced expression of pro-angiogenic factors [28]. Additionally, senescent neural progenitor cells contribute to the development of multiple sclerosis [29]. Specifically, senescent neural progenitor cells inhibit oligodendrocyte differentiation and contribute to demyelination via the production of high mobility group box (HMGB)1, which suppresses oligodendrocyte progenitor cell proliferation and induces pro-inflammatory transcriptomic changes in oligodendrocyte progenitor cells [29]. Thus, senescent stem cells impair wound healing, promote the development of inflammatory diseases and limit the use of stem cells in therapeutic applications.

In addition to the direct effects on stem cells, there are two potential pathways by which senescence may contribute to disease. First, senescent cells stop proliferating, which prevents tissue repair after injury. Second, as senescent cells accumulate, they produce, for unclear reasons, low levels of inflammatory mediators, a phenomenon which has been termed "inflammaging", particularly when evident in older people [30-33]. Senescent cells contribute to chronic inflammation via the senescence-associated secretory 
phenotype (SASP), the collective term for the pro-inflammatory chemokines and cytokines released by senescent cells [34]. The SASP is comprised of a number of pro-inflammatory molecules, including tumour necrosis factor (TNF)- $\alpha$, IL- $1 \alpha / \beta$, IL-6, IL-8, CC-chemokine ligand (CCL)-2 and others [35-38]. The SASP seems to be a double-edged sword. On the one hand, long-term exposure of SASP inflammatory mediators can lead to over-recruitment of damaging immune cells, resulting in chronic inflammatory diseases and even fibrosis $[5,39]$. On the other hand, in the short term, the SASP promotes wound healing that is required to respond to acute cellular damage $[12,13,40]$.

Importantly, the specific characteristics of the SASP may be dependent on the cell type and the mechanism of senescence induction [41]. For example, the SASP associated with fibroblasts contains significantly more SASP factors than the SASP associated with renal epithelial cells [41]. Some SASP pathways are shared between fibroblasts and epithelial cells, while some pathways are distinct to each cell type. Specifically, senescent epithelial cells upregulate pathways associated with protein translation and degradation; senescent fibroblasts upregulate pathways associated with apoptosis, ROS generation and extracellular matrix reorganisation; and both senescent fibroblasts and epithelial cells upregulate pathways associated with vesicle transport, metabolism and detoxification [41]. Note that in this particular study, senescence was induced in vitro in human cell lines. However, the investigators identified several secreted proteins in their in vitro models which were also found in the plasma of older subjects, suggesting that the findings of the study could be translatable in vivo. In vitro senescence is associated with the accumulation of sterile inflammatory mediators, like HMGB1, a nuclear protein that can activate inflammation [41]. Furthermore, SASP mediators such as TNF- $\alpha$ can induce senescence, which probably further enhances the SASP in a feed-forward loop [42]. Thus, one of the mechanisms by which senescence leads to chronic inflammation may be by the release of sterile inflammatory mediators, also known as damage-associated molecular patterns. Additionally, the SASP is temporally dynamic and the dynamics change depending on the cell type [43]. Overall, senescence induces distinct cellular programmes that lead to the secretion of proteins that promote chronic inflammation. Clearly, dissecting the physiological from the pathophysiological aspects of senescence will be required for the development of effective therapies targeting senescence to alleviate age-related diseases.

\section{Senescence-targeted therapeutics}

Recently, there has been a focus on alleviating the negative effects of cellular senescence using senescence-targeted therapeutics. Such therapeutics fall into two primary categories: SASP inhibition and senescent cell removal. SASP inhibition involves downregulating various components of the SASP, or inhibiting the inflammatory pathways that SASP factors engage, with the goal of ameliorating the deleterious effects of SASP factors. This strategy is successful in a variety of contexts. For example, Janus kinase inhibition alleviates inflammation, both locally in the adipose tissue and systemically, and reduces frailty in aged mice [44]. Evidence that the SASP may be related to the pathogenesis of age-related diseases comes from reports that pharmacological tools to inhibit NF- $\kappa \mathrm{B}$ signalling ameliorate age-related diseases. Inhibition of the mammalian target of rapamycin by rapamycin selectively reduces $N F-\kappa B$ signalling (a major inflammation signalling hub) in senescent cells through suppression of IL-1 $\alpha$ activity, reduces age-associated cognitive decline, improves immune function in older people and increases the lifespan of

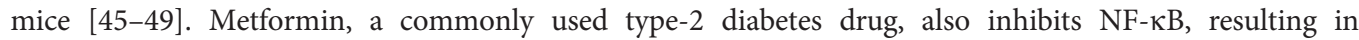
improved lifespan in mice, and reduces all-cause mortality and age-related disease in humans [50-53]. However, inhibiting the SASP results in off-target side-effects including neutrophilia, nephrotoxicity and changes in T-cell phenotype due to the myriad interconnected pathways involved in inflammatory signalling [54]. Specifically, inhibition of the NF- $\mathrm{B}$ pathway, either by genetic deletion or small-molecule inhibition, has been shown to inhibit T-cell, B-cell and lymphoid progenitor cell development [54, 55]; induce apoptosis of thymocytes, B-cells and macrophages [56, 57]; impair lymphocyte growth and cytokine production [58]; and potentially increase susceptibility to various infections [59]. All of these strategies target inflammatory pathways that senescence may activate, but do not specifically target senescent cells.

Removing senescent cells involves directly targeting senescent cells by inducing apoptosis. The first therapeutics designed to directly kill senescent cells (termed "senolytics") targeted the BCL-2 protein family, an anti-apoptotic pathway that is involved in oncogenesis. However, the most widely studied therapeutic strategy is a combination therapy involving the administration of the tyrosine kinase inhibitor dasatinib (D) and the flavonoid quercetin (Q). The "D+Q" combination reduces senescent cell burden and improves healthspan and lifespan in aged mice, reduces vascular stiffening, improves vasomotor function and improves pulmonary function in murine models of pulmonary fibrosis [60-64]. Indeed, preliminary clinical trials have shown evidence that $\mathrm{D}+\mathrm{Q}$ treatment reduces senescent cell burden and may improve physical function in patients with pulmonary fibrosis $[65,66]$. However, we still do not fully appreciate the 
potential off-target effects of senolytic agents such as D+Q, as well as their mechanism of action in humans, which will require elucidation before they can be used to treat age-related diseases.

\section{Cellular senescence in the lung}

In addition to replicative senescence induced by ageing, several other factors contribute to the development of stress-induced senescence in the lung, including radiation therapy, smoking, mechanical ventilation and chronic use of supplemental oxygen (figure 1) [24,67-70], which supports the idea that other processes besides natural ageing lead to senescence. Increasing numbers of senescent cells correlate with increasing concentrations of SASP factors. This is supported by the finding that the cytokines IL- 6 and IL-10 and neutrophil counts are elevated in the bronchoalveolar lavage fluid of older donors (note: "older" is designated age $>65$ years in this review), which may exhibit senescence in the lung [71]. This age-related phenotype correlates with age-related lung diseases such as COPD. Furthermore, patients with COPD exhibit increased concentrations of senescent type II alveolar epithelial cells [72, 73] and lung fibroblasts [74], as well as elevated levels of inflammatory cytokines including IL-1 $\beta$, IL-6, IL-8, IL-10 and CCL-2 [75-78]. While a causal link is difficult to prove, the low-grade inflammation caused by lung senescent cells may contribute to the development of and/or exacerbate COPD. Similar patterns of elevated inflammatory cytokine levels and senescent cells are linked to other chronic lung diseases such as asthma $[79,80]$. Furthermore, the exuberant wound-healing response mediated by SASP factors, combined with the inability to effectively repair tissue due to the cessation of proliferation, and possibly impairments in stem cell functions, may promote fibrosis and contribute to idiopathic pulmonary fibrosis [81-84]. Thus, lung senescent cells probably contribute to the severity of many chronic lung diseases.

The potential contributions of lung cellular senescence in acute respiratory conditions remain unclear. In a murine model of acute lung injury, senescent alveolar macrophages exhibit enhanced activation and secretion of pro-inflammatory cytokines, resulting in enhanced disease severity [85]. Similarly, aged mice, which probably exhibit increased lung senescence, exhibit enhanced lung permeability and increased levels of ROS in response to a lipopolysaccharide challenge, which is linked to deficient Nox4 (a ROS-generating mitochondrial protein) ubiquination [86]. Additionally, older trauma patients are significantly more likely to develop acute respiratory distress syndrome (ARDS), and older patients with ARDS exhibit a higher mortality rate [87]. However, in all of these studies the pathological role of senescence was not tested. Additionally, a recent study found that lung cellular senescence protects against lung injury induced by mechanical ventilation by preventing apoptosis [68]. Clearly, the impact of cellular senescence on acute lung conditions requires future investigation.

\section{Cellular senescence and respiratory viral infections}

The coronavirus disease 2019 (COVID-19) pandemic continues to claim thousands of lives every day and disproportionately affects older people. Given this, how cellular senescence impacts the host response to acute respiratory viral infections is particularly relevant. Older people experience increased susceptibility to respiratory viruses such as influenza, respiratory syncytial virus (RSV), SARS and now SARS-CoV-2 [88-92]. With COVID-19, the disease that results from SARS-CoV-2 infection, older people are $\sim 20$-fold more likely to die than younger people [93]. This effect has been partially attributed to defects associated with the ageing of the immune system, termed "immunosenescence", which is distinct from classical cellular senescence as defined earlier and reviewed elsewhere [94-96]. Briefly, immunosenescence has

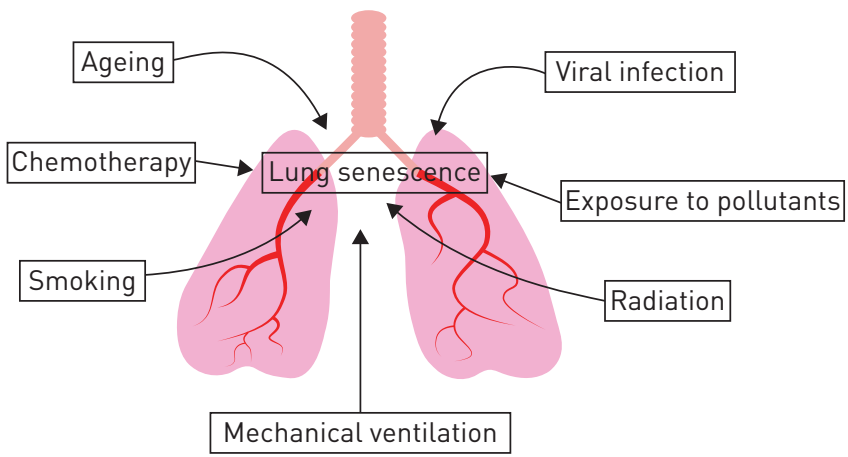

FIGURE 1 Causes of lung senescence. Many factors contribute to the induction of senescence within lung cells. Apart from natural ageing, other sources of senescence include chronic exposure to pollutants and cigarette smoke, chemotherapy, ionising radiation, respiratory viral infections and the use of mechanical ventilators. Each of these sources may induce senescence to varying degrees, and it remains unclear whether senescence originating from different sources and in different cells contributes to pathology similarly or differently. 
pleiotropic effects on the immune system including 1) a decrease in the proliferative capacity of haematopoietic stem cells; 2) dysregulation of innate immunity; 3 ) reduced numbers of naïve T-cells with thymic involution; 4) accumulation of memory T- and B-cells; and 5) a general decline in both T- and B-cell function [94, 97-99]. Specifically, both $\mathrm{CD}^{+}$and $\mathrm{CD}^{+}$T-cells from aged hosts (e.g. rodents, humans) exhibit a reduced proliferative capacity associated with downregulation of surface costimulatory receptors, CD27 and CD28, which is accompanied by shortened telomeres. Collectively, these alterations within the immune system with ageing result in an impaired ability to fight viral infections and lead to reduced vaccine efficiency [90]. In addition to natural ageing, chronic viral infections (e.g. HIV, cytomegalovirus infection) induce a phenotype of reduced T-cell function (i.e. reduced proliferation and cytokine secretion) that shares features with immunosenescence [100, 101]. Although ageing of the immune system impairs host defence to acute respiratory viral infections [90], the role that cellular senescence of lung cells plays in the pathophysiology of acute respiratory viral infections is not well understood. Clearly, older people are highly susceptible to viral infections due to myriad effects exerted by ageing on the immune system. But how much of this is contributed by cellular senescence, as defined earlier, remains to be elucidated. Indeed, it is not clear whether cellular senescence, due to natural ageing or due to an age-independent stressor, promotes or prevents acute respiratory viral infections. To the best of our knowledge, there are only a few studies on the role of cellular senescence and acute respiratory viral infections, with some showing contrasting results.

There are studies that claim that cellular senescence enhances antiviral immunity [102], which is biologically plausible, as SASP factors, which includes chemokines (e.g. CxCL1/2) that recruit innate immune cells like neutrophils, may augment host defence to viral infections [103]. Specifically, one study showed that cellular senescence inhibits vesicular stomatis virus (an RNA virus) replication in both primary murine fibroblasts with replication-induced senescence and in a tumour cell line with chemotherapy-induced senescence [102], possibly due to SASP factors, including the release of type I interferons (IFN), important antiviral cytokines [104]. Additionally, mice that were induced to exhibit senescence within the lung via bleomycin exposure were more resistant to vesicular stomatis virus infection in vivo than mice that were not exposed to bleomycin. This is compatible with in vitro studies that found that type I IFN, in addition to other SASP components such as IL-6 and IL-8, induces cellular senescence $[105,106]$, and vice versa. Additionally, cellular fusion caused by the fusogenic measles virus induces cellular senescence, including the release of SASP factors, in fibroblasts in vitro [107], although the in vivo implications of this finding are unclear. Collectively, these studies suggest that senescence that is induced acutely by viruses may be a part of the body's antiviral immune response [108, 109]. This defence mechanism may have evolved to allow virus-infected cells to secrete SASP factors to limit viral replication in neighbouring cells and ultimately viral spread throughout the lung. Furthermore, the SASP factors may enhance host defence by promoting immune cell recruitment to the lung. But if these SASP factors are produced exuberantly, immune pathology could ensue (figure 2). Finally, some viruses, including human papillomavirus and hepatitis B, have evolved machinery specifically to overcome cellular senescence [110, 111 , suggesting that senescence plays a role in antiviral defence in some contexts.

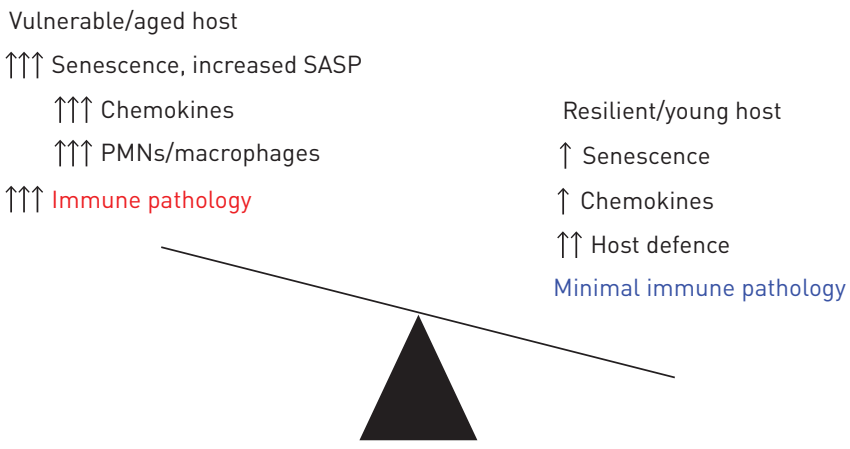

Lung cellular senescence and respiratory viral infections

FIGURE 2 Hypothesis: senescence may play divergent roles during acute respiratory viral infections depending on host resilience. In young hosts, senescence induced by viral infection may play a positive role by recruiting neutrophils (PMNs) and other immune cells via the senescence-associated secretory phenotype (SASP), resulting in viral clearance and tissue repair. However, in aged or vulnerable hosts, senescence induced by viral infection, in addition to the senescence already present, may tip the balance towards pathology. This pathology is characterised by an exuberant immune response, including high levels of SASP cytokines and chemokines, causing exaggerated recruitment of PMNs and other immune cells, resulting in lung tissue damage. 
However, there is evidence that senescence contributes to the pathophysiology of respiratory viral infections. RSV induces DNA damage resulting in senescence in both mononuclear cells and lung epithelia in young mice [25], which enhances airway tissue remodelling (exhibited by a loss of ciliated cells and an increase in secretory cells [112]), potentially leading to permanent tissue damage and fibrosis. Furthermore, senescent cells may promote viral replication. For example, the influenza virus replicates more efficiently within senescent human bronchial epithelial cells than in non-senescent cells [113]. Similar results are found with varicella zoster virus, a herpes virus, which replicates more efficiently in senescent human dermal fibroblasts than nonsenescent cells [113]. A potential explanation for this phenomenon may be that senescence induced by these viruses downregulates the antiviral type I IFN programme, including key signalling proteins, e.g. STING and interferon regulatory factor-3, upon infection in vitro with varicella virus [113]. This downregulation could lead to reduced secretion of antiviral IFNs, promoting viral replication. However, it is not known whether this occurs in vivo. Differences between this study and the contrasting one discussed earlier, which found that senescence reduces replication of vesicular stomatis virus [102], could be due to the different viruses employed (i.e. vesicular stomatitis versus influenza, varicella), different cell types (MCF7, A549 cell lines and primary murine embryo fibroblast cells in the vesicular stomatitis study versus primary human bronchial epithelial cells and primary human dermal fibroblasts in the influenza/varicella study), and different approaches to induce senescence (replicative senescence and bleomycin in the vesicular stomatitis study versus only replicative senescence in the influenza/varicella study). Clearly, future studies are required to determine if senescence is harmful or beneficial to pathologically relevant respiratory viruses.

\section{Cellular senescence: friend or foe in respiratory viral infections?}

The overall impact of cellular senescence on acute respiratory viruses may depend on host resilience factors (figure 2). For example, the SASP may have evolved to enhance host defence by increasing the recruitment of immune cells. This is probably beneficial in resilient young hosts who do not overproduce SASP factors. Indeed, initial neutrophil recruitment to the lung is critical to host defence to influenza infection [103]. But in vulnerable aged hosts, the SASP factors may be produced at pathologically high levels to promote immune pathology. Specifically, CXCL-1, CXCL-2 and IL-17 are increased in the lungs of aged mice even before viral infection, and accompany excessive neutrophil recruitment into the lung to enhance mortality during acute influenza infection [103]. In this study, senescent alveolar epithelial cells were found to produce more neutrophil chemoattractants and thus increase neutrophil chemotaxis. Similarly, aged rhesus macaques express higher levels of CCL-2, IL-6 and IL-8 in the lungs in response to influenza infection, suggesting an exaggerated innate immune response [114]. Based on these studies, inhibiting the SASP or reducing lung senescent cells might be beneficial in aged hosts infected with acute respiratory viral infections. However, formal investigation is required to determine whether removing senescent cells prior to infection is sufficient, or if treatment during infection is required. Importantly, vulnerable hosts, including older people and people with comorbidities such as diabetes, are more susceptible to SARS-CoV-2, which emerging studies suggest is due to the cytokine storm and excessive neutrophil effector functions within the lung [90].

We still do not know whether senescent cells in the lung ultimately increase or reduce viral replication. Respiratory viruses may "hijack" the senescent machinery within the infected cell to downregulate the IFN pathway to promote viral packaging and ultimately enhance viral replication within the cell. It is not clear yet if this occurs in vivo and, if so, what underlying mechanisms are involved. In contrast, activation of the senescence machinery within a virally infected cell may be a damage-response mechanism that leads to the secretion of antiviral cytokines to limit viral replication, an issue that also requires future investigation. Alternatively, senescence may simply be a side-effect of viral infection; during and after infection, various inflammatory cytokines are upregulated, resulting in the induction of senescence in infected cells.

\section{Conclusions}

Ultimately, the impact of senescence on respiratory viral infections requires further investigation before firm conclusions can be drawn. The impact of senescence on both viral replication and mortality in respiratory viral infections such as influenza, RSV, SARS and SARS-CoV-2 should be studied comprehensively to determine if senescence-targeted therapies, such as senolytics, might be effective, or not, at reducing the age-dependent mortality of acute respiratory viral infections. Furthermore, it remains unclear whether senescence associated with natural ageing results in the same impact on respiratory viral infections as stress-induced senescence; thus, a direct comparison between such models of senescence is warranted. For example, do mice with bleomycin-induced lung senescence exhibit a similar phenotype and mortality response to aged mice when infected with influenza, RSV or other respiratory viral infections?

Additionally, the effectiveness (or lack thereof) of senescence-targeted therapies broadly, and senolytics, specifically, has yet to be assessed during respiratory viral infections. While senolytics have shown some 
therapeutic efficacy in pre-clinical models of chronic illnesses such as pulmonary fibrosis, it is unknown if these effects translate to more acute conditions, and if so, how the therapeutic regimen might be optimised. For example, it may be that prophylactic senolytic treatment is effective at reducing the total senescent cell concentration in the lungs, thereby improving disease outcomes, while post-infection treatment may not be as effective, especially if removing senescent cells impairs wound healing and reduces inflammation resolution. Examining these outstanding questions will shed light on how senescence impacts our host defence to respiratory viruses, which will continue to disproportionately impact older people for the foreseeable future, particularly during the current COVID-19 pandemic. Thus, moving forward, significant research efforts focused on the interactions between cellular senescence and respiratory viral infections are clearly warranted.

Author contributions: All authors were involved in the design of the review. W.J. Kelley wrote the first draft, which was then edited by D.R. Goldstein and R.L. Zemans. All authors approved the final manuscript.

Conflict of interest: None declared.

Support Statement: W.J. Kelley is supported by the National Institutes of Health Training Grant T32HL007622. D.R. Goldstein is supported by NIH awards: R01AG028082, R01HL127687, R01AI13834, K07AG050096 and T32AG062403. R.L. Zemans is supported by NIH awards: R01HL147920, R01HL131608 and R01AG028082. Funding information for this article has been deposited with the Crossref Funder Registry.

\section{References}

Hayflick L, Moorhead PS. The serial cultivation of human diploid cell strains. Exp Cell Res 1961; 25: 585-621.

Hayflick L. The limited in vitro lifetime of human diploid cell strains. Exp Cell Res 1965; 37: 614-636.

Dimri GP, Lee X, Basile G, et al. A biomarker that identifies senescent human cells in culture and in aging skin in vivo. Proc Natl Acad Sci USA 1995; 92: 9363-9367.

4 Morty RE, Prakash YS. Senescence in the lung: is this getting old? Am J Physiol Lung Cell Mol Physiol 2019; 316: L822-L825.

$5 \quad$ He S, Sharpless NE. Senescence in health and disease. Cell 2017; 169: 1000-1011.

6 Childs BG, Durik M, Baker DJ, et al. Cellular senescence in aging and age-related disease: from mechanisms to therapy. Nat Med 2015; 21: 1424-1435.

Campisi J. Aging, cellular senescence, and cancer. Annu Rev Physiol 2013; 75: 685-705.

Sager R. Senescence as a mode of tumor suppression. Environ Health Perspect 1991; 93: 59-62.

Braig M, Lee S, Loddenkemper C, et al. Oncogene-induced senescence as an initial barrier in lymphoma development. Nature 2005; 436: 660-665.

10 Chen Z, Trotman LC, Shaffer D, et al. Crucial role of p53-dependent cellular senescence in suppression of Pten-deficient tumorigenesis. Nature 2005; 436: 725-730.

11 Schmitt CA, Fridman JS, Yang M, et al. A senescence program controlled by p53 and p16INK4a contributes to the outcome of cancer therapy. Cell 2002; 109: 335-346.

12 Demaria M, Ohtani N, Youssef SA, et al. An essential role for senescent cells in optimal wound healing through secretion of PDGF-AA. Dev Cell 2014; 31: 722-733.

13 Krizhanovsky V, Yon M, Dickins RA, et al. Senescence of activated stellate cells limits liver fibrosis. Cell 2008; 134: 657-667.

14 Kim K-H, Chen C-C, Monzon RI, et al. Matricellular protein CCN1 promotes regression of liver fibrosis through induction of cellular senescence in hepatic myofibroblasts. Mol Cell Biol 2013; 33: 2078-2090.

15 Fitzner B, Müller S, Walther M, et al. Senescence determines the fate of activated rat pancreatic stellate cells. J Cell Mol Med 2012; 16: 2620-2630.

16 Jun JI, Lau LF. The matricellular protein CCN1 induces fibroblast senescence and restricts fibrosis in cutaneous wound healing. Nat Cell Biol 2010; 12: 676-685.

17 Muñoz-Espín D, Cañamero M, Maraver A, et al. Programmed cell senescence during mammalian embryonic development. Cell 2013; 155: 1104-1118.

18 Storer M, Mas A, Robert-Moreno A, et al. Senescence is a developmental mechanism that contributes to embryonic growth and patterning. Cell 2013; 155: 1119-1130.

19 McHugh D, Gil J. Senescence and aging: causes, consequences, and therapeutic avenues. J Cell Biol 2018; 217: 65-77.

20 López-Otín C, Blasco MA, Partridge L, et al. The hallmarks of aging. Cell 2013; 153: 1194-1217.

21 Ksiazek K, Mikula-Pietrasik J, Olijslagers S, et al. Vulnerability to oxidative stress and different patterns of senescence in human peritoneal mesothelial cell strains. Am J Physiol Regul Integr Comp Physiol 2009; 296: 374-382.

22 Weyemi U, Lagente-Chevallier O, Boufraqech $\mathrm{M}$, et al. ROS-generating NADPH oxidase NOX4 is a critical mediator in oncogenic H-Ras-induced DNA damage and subsequent senescence. Oncogene 2012; 31: 1117-1129. van Deursen JM. The role of senescent cells in ageing. Nature 2014; 509: 439-446.

24 Nyunoya T, Monick MM, Klingelhutz A, et al. Cigarette smoke induces cellular senescence. Am J Respir Cell Mol Biol 2006; 35: 681-688.

25 Martínez I, García-Carpizo V, Guijarro T, et al. Induction of DNA double-strand breaks and cellular senescence by human respiratory syncytial virus. Virulence 2016; 7: 427-442.

26 Wheaton K, Campuzano D, Ma W, et al. Progerin-induced replication stress facilitates premature senescence in Hutchinson-Gilford progeria syndrome. Mol Cell Biol 2017; 37: e00659-16.

27 Gnani D, Crippa S, della Volpe L, et al. An early-senescence state in aged mesenchymal stromal cells contributes to hematopoietic stem and progenitor cell clonogenic impairment through the activation of a pro-inflammatory program. Aging Cell 2019; 18: e12933. 
Liu J, Ding Y, Liu Z, et al. Senescence in mesenchymal stem cells: functional alterations, molecular mechanisms, and rejuvenation strategies. Front Cell Dev Biol 2020; 8: 258.

Nicaise AM, Wagstaff LJ, Willis CM, et al. Cellular senescence in progenitor cells contributes to diminished remyelination potential in progressive multiple sclerosis. Proc Natl Acad Sci USA 2019; 116: 9030-9039.

Sanada F, Taniyama Y, Muratsu J, et al. Source of chronic inflammation in aging. Front Cardiovasc Med 2018; 5: $1-5$.

Baylis D, Bartlett DB, Patel HP, et al. Understanding how we age: insights into inflammaging. Longev Healthspan 2013; $2: 8$.

Franceschi C, Capri M, Monti D, et al. Inflammaging and anti-inflammaging: a systemic perspective on aging and longevity emerged from studies in humans. Mech Ageing Dev 2007; 128: 92-105.

Piber D, Olmstead R, Cho JHJ, et al. Inflammaging: age and systemic, cellular, and nuclear inflammatory biology in older adults. J Gerontol A Biol Sci Med Sci 2019; 74: 1716-1724.

Campisi J. Aging, cellular senescence, and cancer. Annu Rev Physiol 2013; 75: 685-705.

Tchkonia T, Zhu Y, van Deursen J, et al. Cellular senescence and the senescent secretory phenotype: therapeutic opportunities. J Clin Invest 2013; 123: 966-972.

Freund A, Orjalo AV, Desprez PY, et al. Inflammatory networks during cellular senescence: causes and consequences. Trends Mol Med 2010; 16: 238-246.

Coppé JP, Patil CK, Rodier F, et al. Senescence-associated secretory phenotypes reveal cell-nonautonomous functions of oncogenic RAS and the p53 tumor suppressor. PLoS Biol 2008; 6: 2853-2868.

Kumar S, Millis AJT, Baglioni C. Expression of interleukin 1-inducible genes and production of interleukin 1 by aging human fibroblasts. Proc Natl Acad Sci USA 1992; 89: 4683-4687.

Childs BG, Durik M, Baker DJ, et al. Cellular senescence in aging and age-related disease: from mechanisms to therapy. Nat Med 2015; 21: 1424-1435.

Xue W, Zender L, Miething C, et al. Senescence and tumour clearance is triggered by p53 restoration in murine liver carcinomas. Nature 2007; 445: 656-660.

Basisty $\mathrm{N}$, Kale A, Jeon $\mathrm{OH}$, et al. A proteomic atlas of senescence-associated secretomes for aging biomarker development. PLoS Biol 2020; 18: e3000599.

Desdín-Micó G, Soto-Heredero G, Aranda JF, et al. T cells with dysfunctional mitochondria induce multimorbidity and premature senescence. Science 2020; 368: 1371-1376.

Hernandez-Segura A, de Jong T V, Melov S, et al. Unmasking transcriptional heterogeneity in senescent cells. Curr Biol 2017; 27: 2652-2660.

$\mathrm{Xu}$ M, Tchkonia T, Ding H, et al. JAK inhibition alleviates the cellular senescence-associated secretory phenotype and frailty in old age. Proc Natl Acad Sci USA 2015; 112: E6301-E6310.

Wilkinson JE, Burmeister L, Brooks S V, et al. Rapamycin slows aging in mice. Aging Cell 2012; 11: 675-682.

Majumder S, Caccamo A, Medina DX, et al. Lifelong rapamycin administration ameliorates age-dependent cognitive deficits by reducing IL-1 $\beta$ and enhancing NMDA signaling. Aging Cell 2012; 11: 326-335.

Mannick JB, Del Giudice G, Lattanzi M, et al. mTOR inhibition improves immune function in the elderly. Sci Transl Med 2014; 6: 268ra179.

Laberge RM, Sun Y, Orjalo A V, et al. MTOR regulates the pro-tumorigenic senescence-associated secretory phenotype by promoting IL1A translation. Nat Cell Biol 2015; 17: 1049-1061.

Mannick JB, Morris M, Hockey HU, et al. TORC1 inhibition enhances immune function and reduces infections in the elderly. Sci Transl Med 2018; 10: eaaq1564.

Moiseeva O, Deschênes-Simard X, St-Germain E, et al. Metformin inhibits the senescence-associated secretory phenotype by interfering with IKK/NF-KB activation. Aging Cell 2013; 12: 489-498.

Martin-Montalvo A, Mercken EM, Mitchell SJ, et al. Metformin improves healthspan and lifespan in mice. Nat Commun 2014; 4: 2192.

Campbell JM, Bellman SM, Stephenson MD, et al. Metformin reduces all-cause mortality and diseases of ageing independent of its effect on diabetes control: a systematic review and meta-analysis. Ageing Res Rev 2017; 40: 31-44.

Bannister CA, Holden SE, Jenkins-Jones S, et al. Can people with type 2 diabetes live longer than those without? A comparison of mortality in people initiated with metformin or sulphonylurea monotherapy and matched, non-diabetic controls. Diabetes Obes Metab 2014; 16: 1165-1173.

Zhang Q, Lenardo MJ, Baltimore D. 30 years of NF-KB: a blossoming of relevance to human pathobiology. Cell 2017; 168: 37-57.

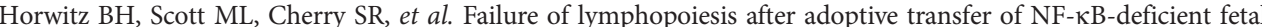
liver cells. Immunity 1997; 6: 765-772.

Nagashima K, Sasseville VG, Wen D, et al. Rapid TNFR1-dependent lymphocyte depletion in vivo with a selective chemical inhibitor of IKK $\beta$. Blood 2006; 107: 4266-4273. apoptosis - CREB and NF- $\mathrm{KB}$ as key regulators. Immunity 2005; 23: 319-329.

Schmidt-Supprian M, Tian J, Ji H, et al. IкB kinase 2 deficiency in T cells leads to defects in priming, B cell help, germinal center reactions, and homeostatic expansion. J Immunol 2004; 173: 1612-1619.

Lavon I, Goldberg I, Amit S, et al. High susceptibility to bacterial infection, but no liver dysfunction, in mice compromised for hepatocyte NF-кB activation. Nat Med 2000; 6: 573-577.

Zhu Y, Tchkonia T, Pirtskhalava T, et al. The Achilles' heel of senescent cells: from transcriptome to senolytic drugs. Aging Cell 2015; 14: 644-658.

Roos CM, Zhang B, Palmer AK, et al. Chronic senolytic treatment alleviates established vasomotor dysfunction in aged or atherosclerotic mice. Aging Cell 2016; 15: 973-977.

Xu M, Pirtskhalava T, Farr JN, et al. Senolytics improve physical function and increase lifespan in old age. Nat Med 2018; 24: 1246-1256.

Lehmann M, Korfei M, Mutze K, et al. Senolytic drugs target alveolar epithelial cell function and attenuate experimental lung fibrosis ex vivo. Eur Respir J 2017; 50: 1602367.

Schafer MJ, White TA, Iijima K, et al. Cellular senescence mediates fibrotic pulmonary disease. Nat Commun 2017; 8: 14532. 

first-in-human, open-label, pilot study. EBioMedicine 2019; 40: 554-563. report from a clinical trial of dasatinib plus quercetin in individuals with diabetic kidney disease. EBioMedicine 2019; 47: 446-456.

67 Ahmad T, Sundar IK, Lerner CA, et al. Impaired mitophagy leads to cigarette smoke stress-induced cellular senescence: implications for chronic obstructive pulmonary disease. FASEB J 2015; 29: 2912-2929.

68 Blázquez-Prieto J, Huidobro C, López-Alonso I, et al. Activation of p21 limits acute lung injury and induces early senescence after acid aspiration and mechanical ventilation. bioRxiv 2020; preprint [https://doi.org/10.1101/ 2020.03.24.005983].

69 Parikh P, Britt RD, Manlove LJ, et al. Hyperoxia-induced cellular senescence in fetal airway smooth muscle cells. Am J Respir Cell Mol Biol 2019; 61: 51-60.

70 Klimova TA, Bell EL, Shroff EH, et al. Hyperoxia-induced premature senescence requires p53 and pRb, but not mitochondrial matrix ROS. FASEB J 2009; 23: 783-794.

71 Meyer KC, Ershler W, Rosenthal NS, et al. Immune dysregulation in the aging human lung. Am J Respir Crit Care Med 1996; 153: 1072-1079.

72 Tsuji T, Aoshiba K, Nagai A. Alveolar cell senescence exacerbates pulmonary inflammation in patients with chronic obstructive pulmonary disease. Respiration 2010; 80: 59-70.

73 Zhou F, Onizawa S, Nagai A, et al. Epithelial cell senescence impairs repair process and exacerbates inflammation after airway injury. Respir Res 2011; 12: 78.

74 Müller KC, Welker L, Paasch K, et al. Lung fibroblasts from patients with emphysema show markers of senescence in vitro. Respir Res 2006; 7: 32.

75 Traves SL, Culpitt S V, Russell REK, et al. Increased levels of the chemokines GRO $\alpha$ and MCP-1 in sputum samples from patients with COPD. Thorax 2002; 57: 590-595.

76 De Boer WI, Sont JK, Van Schadewijk A, et al. Monocyte chemoattractant protein I, interleukin 8, and chronic airways inflammation in COPD. J Pathol 2000; 190: 619-626.

77 Gessner C, Scheibe R, Wötzel M, et al. Exhaled breath condensate cytokine patterns in chronic obstructive pulmonary disease. Respir Med 2005; 99: 1229-1240.

78 Aldera JK, Barkauskas CE, Limjunyawong N, et al. Telomere dysfunction causes alveolar stem cell failure Proc Natl Acad Sci USA 2015; 112: 5099-5104.

79 Busse PJ, Birmingham JM, Calatroni A, et al. Effect of aging on sputum inflammation and asthma control. J Allergy Clin Immunol 2017; 139: 1808-1818.

80 Hawkins GA, Robinson MB, Hastie AT, et al. IL6R variation Asp(358)Ala is a potential modifier of lung function in asthma. J Allergy Clin Immunol 2012; 130: 510-515.

81 Kuwano K, Kunitake R, Kawasaki M, et al. P21Waf1/Cip1/Sdi1 and p53 expression in association with DNA strand breaks in idiopathic pulmonary fibrosis. Am J Respir Crit Care Med 1996; 154: 477-483.

82 Lomas NJ, Watts KL, Akram KM, et al. Idiopathic pulmonary fibrosis: immunohistochemical analysis provides fresh insights into lung tissue remodelling with implications for novel prognostic markers. Int J Clin Exp Pathol 2012; 5: 58-71.

83 Álvarez D, Cárdenes N, Sellarés J, et al. IPF lung fibroblasts have a senescent phenotype. Am J Physiol Lung Cell Mol Physiol 2017; 313: L1164-L1173.

84 Habermann A, Gutierrez A, Bui L, et al. Single-cell RNA sequencing reveals profibrotic roles of distinct epithelial and mesenchymal lineages in pulmonary fibrosis. Sci Adv 2020; 6: eaba1972.

85 Brandenberger $\mathrm{C}$, Kling KM, Vital M, et al. The role of pulmonary and systemic immunosenescence in acute lung injury. Aging Dis 2018; 9: 553-565.

86 Palumbo S, Shin YJ, Ahmad K, et al. Dysregulated Nox4 ubiquitination contributes to redox imbalance and age-related severity of acute lung injury. Am J Physiol Lung Cell Mol Physiol 2017; 312: L297-L308.

87 Johnston CJ, Rubenfeld GD, Hudson LD. Effect of age on the development of ARDS in trauma patients. Chest 2003; 124: 653-659.

88 Falsey AR, Walsh EE. Respiratory syncytial virus infection in elderly adults. Drugs Aging 2005; 22: 577-587.

89 Pop-Vicas A, Gravenstein S. Influenza in the elderly - a mini-review. Gerontology 2011; 57: 397-404.

90 Chen J, Kelley WJ, Goldstein DR. Role of aging and the immune response to respiratory viral infections: potential implications for COVID-19. J Immunol 2020; 205: 313-320.

91 Baas T, Roberts A, Teal TH, et al. Genomic analysis reveals age-dependent innate immune responses to severe acute respiratory syndrome coronavirus. J Virol 2008; 82: 9465-9476.

92 Onder G, Rezza G, Brusaferro S. Case-fatality rate and characteristics of patients dying in relation to COVID-19 in Italy. JAMA 2020; 323: 1775-1776.

93 Zhou F, Yu T, Du R, et al. Clinical course and risk factors for mortality of adult inpatients with COVID-19 in Wuhan, China: a retrospective cohort study. Lancet 2020; 395: 1054-1062.

94 Chou JP, Effros RB. T cell replicative senescence in human aging. Curr Pharm Des 2013; 19: 1680-1698.

95 Shaw AC, Joshi S, Greenwood H, et al. Aging of the innate immune system. Curr Opin Immunol 2010; 22: 507-513.

96 Dorrington MG, Bowdish DME. Immunosenescence and novel vaccination strategies for the elderly. Front Immunol 2013; 4: 171.

97 Aiello A, Farzaneh F, Candore G, et al. Immunosenescence and its hallmarks: how to oppose aging strategically? A review of potential options for therapeutic intervention. Front Immunol 2019; 10: 2247.

98 Nikolich-Žugich J. The twilight of immunity: emerging concepts in aging of the immune system. Nat Immunol 2018; 19: 10-19.

99 Barbé-Tuana F, Funchal G, Schmitz CRR, et al. The interplay between immunosenescence and age-related diseases. Semin Immunopathol 2020; in press [https://doi.org/10.1007/s00281-020-00806-z].

100 Appay V, Almeida JR, Sauce D, et al. Accelerated immune senescence and HIV-1 infection. Exp Gerontol 2007; 42: 432-437.

101 Voehringer D, Blaser C, Brawand P, et al. Viral infections induce abundant numbers of senescent CD8 T cells. J Immunol 2001; 167: 4838-4843. 
Baz-Martínez M, Da Silva-Álvarez S, Rodríguez E, et al. Cell senescence is an antiviral defense mechanism. Sci Rep 2016; 6: 37007.

103 Kulkarni U, Zemans RL, Smith CA, et al. Excessive neutrophil levels in the lung underlie the age-associated increase in influenza mortality. Mucosal Immunol 2019; 12: 545-554.

104 Ivashkiv LB, Donlin LT. Regulation of type I interferon responses. Nat Rev Immunol 2014; 14: 36-49.

105 Kuilman T, Michaloglou C, Vredeveld LCW, et al. Oncogene-induced senescence relayed by an interleukin-dependent inflammatory network. Cell 2008; 133: 1019-1031.

106 Ritschka B, Storer M, Mas A, et al. The senescence-associated secretory phenotype induces cellular plasticity and tissue regeneration. Genes Dev 2017; 31: 172-183.

107 Chuprin A, Gal H, Biron-Shental T, et al. Cell fusion induced by ERVWE1 or measles virus causes cellular senescence. Genes Dev 2013; 27: 2356-2366.

108 Moiseeva O, Mallette FA, Mukhopadhyay UK, et al. DNA damage signaling and p53-dependent senescence after prolonged $\beta$-interferon stimulation. Mol Biol Cell 2006; 17: 1583-1592.

109 Yu Q, Katlinskaya YV, Carbone CJ, et al. DNA-damage-induced type I interferon promotes senescence and inhibits stem cell function. Cell Rep 2015; 11: 785-797.

110 Oishi N, Shilagardi K, Nakamoto Y, et al. Hepatitis B virus X protein overcomes oncogenic RAS-induced senescence in human immortalized cells. Cancer Sci 2007; 98: 1540-1548.

111 DiPaolo JA, Popescu NC, Alvarez L, et al. Cellular and molecular alterations in human epithelial cells transformed by recombinant human papillomavirus. Crit Rev Oncog 1993; 4: 337-360.

112 Persson BD, Jaffe AB, Fearns R, et al. Respiratory syncytial virus can infect basal cells and alter human airway epithelial differentiation. PLoS One 2014; 9: e102368.

113 Kim JA, Seong RK, Shin OS. Enhanced viral replication by cellular replicative senescence. Immune Netw 2016; 16: $286-295$.

114 Josset L, Engelmann F, Haberthur K, et al. Increased viral loads and exacerbated innate host responses in aged macaques infected with the 2009 pandemic H1N1 influenza A virus. J Virol 2012; 86: 11115-11127. 